\title{
OFDM BASED RADCOM SYSTEM WITH IMPROVED Performance Using Digital Beamforming TECHNIQUE
}

\author{
A.Suban ${ }^{1}$, G.Thanga Balaji ${ }^{2}$, H.Mani Aravinth ${ }^{2}$ and C.Kaleeswaran ${ }^{2}$ \\ ${ }^{1}$ Assistant Professor, ${ }^{2}$ UG Final Year Students \\ Velammal College of Engineering and Technology, Madurai, India.
}

\begin{abstract}
In recent past the influence of Radar has played a significant part in various fields. Radar sensing is one of the prime application by which velocity and distance of a moving target can be found out. A joint RadCom system to serve both radar sensing and wireless communication is proposed which ensures better performance in terms of spectral efficiency, extended detection range and cost effectiveness. Such systems demand for a common waveform which is designed in this work that perfectly matches to the system requirements. OFDM multi carrier technique is chosen to generate a common waveform. Applicability of multiple antenna technique for direction of arrival estimation is also considered. MIMO-OFDM technique has gained much interest in the field of communication which improves the signal to noise ratio and lowers the bit error rate. On the other hand the usage of MIMO reflects in the form of interference between signals. In order to overcome this effect beamforming technique is used. In addition to theoretical explanations we have also simulated and discussed the results for the proposed RadCom system using MATLAB simulation tool.
\end{abstract}

\section{KEYWORDS}

RadCom, OFDM, MIMO, DBF, Doppler Effect, Radar Processing.

\section{I.INTRODUCTION}

Radar operations involve radio frequency waves which is being transmitted, reflected from the specified target and received at the receiver. The reflected wave will have information about the target in terms of velocity with which it is moving, distance from the receiver, etc. It undergoes Doppler shift which might result in loss of information in few cases, hence it's noted that Doppler processing is taken into account for such systems. Radar communication can be taken to the next stage by merging wireless communication to it. This provides a unique platform which would offer sharing of radar images from various sources through wireless communication. RadCom systems will have more applications than simple radar or a wireless communication link. Since both the system shares the information mutually, it can be used in areas where there is a demand for high range detection and quick information transfer such as military applications, aeronautical, etc. The prime consideration in combining radar and wireless communication is the need for common waveform that would be suitable for both the operations. Radar sensing requires waveforms with certain property; auto-correlation is one of the foremost properties that it should posses. Linear frequency modulated (LFM) waveforms satisfy this property better than

DOI : $10.5121 /$ ijcsity.2014.2203 
International Journal of Computational Science and Information Technology (IJCSITY) Vol.2, No.2, May 2014 any other waveforms and act as a good candidate for radar processing [1]. The encoding of data can also be done with the LFM signals [2].But the disadvantage of using it is that its symbol rate varies respectively with the chirp rate which would result in lower symbol rate at same bandwidth for a dedicated link[3]. Hence the usage of FM wave will result in poor performance in terms of communication. For good performance in communication link, a CW (continuous wave) will serve the requirement better. Single carrier and multi carrier are two main approaches in a digital communication link. As multi carrier waveforms have great advantages over single carrier systems it's preferred over single carrier singles [4]. OFDM signals serves as a perfect candidate matching to the system requirements [5]. The waveform design should satisfy all the parameters taken into account including the bandwidth of the system. Doppler shift is another factor that we should look into [8]. Its acts as a deteriorating factor in information transfer. To find the angle of arrival i.e. the exact 2-D location of the target Digital Beam Forming technique is used [14]. It's a multiple antenna technique similar to MIMO that we use for wireless communication. MIMO and DBF are similar in few aspects that it uses multiple array antennas with baseband conversion and sampling of signals from individual antennas, both apply fully digital signal processing. The entire operation is carried out in 24GhzISM band .Since its license free and satisfy the minimum bandwidth requirement it is perfectly suitable for the proposed RADCOM system.

\section{II.WAVEFORM DESIGN}

The main challenge in establishing a RADCOM system is to identify a suitable waveform for both the joint operation. In general a radar system makes use of continuous waveforms with optimum auto correlation properties. Radar waveforms generally follow pulse compression technique in which the waveform is modulated and correlation processing is applied to it. The waveform in our case is frequency modulated and it can be either linear or non-linear. Frequency modulated continuous wave (FMCW) is the most commonly used waveform for radar sensing applications. In case of wireless communication the system should take care of two main parameters the bit error rate and data rate. In order to get good bit error rate and data rate we go far OFDM multi carrier rather than single carrier signal. Discrete modulation techniques such as phase shift keying (PSK) or quadrature amplitude modulation (QAM) is carried out in recent digital communications [5]. It applies perfectly for BPSK and QPSK signaling since it can withstand Doppler and fading. Phase coded waveforms like barker codes could also contribute to the waveform design [6]. In a single carrier signal they have used DSSS (direct sequence spread spectrum) in earlier stages of communications, similar to this in radar we can go for $\mathrm{m}$-sequence radar system which would be result from the discrete modulation. Thus waveforms with discrete modulation will be used for a joint radar sensing and wireless communication system. In contrast to single-carrier waveforms for OFDM transmission generally a rectangular pulse shape is applied for the individual subcarriers. The guard interval or cyclic prefix, which is a partial cyclic repetition of the time domain signal allows for fully compensating multipath propagation effects. Hence an OFDM multicarrier waveform is used to transmit the arbitrary data with the suitable channel coding techniques.

\section{III.WIRELESS COMMUNICATION}

\section{A. Multiple antenna technique}

In a dedicated wireless communication system in order to increase the signal to noise ratio multiple antenna techniques are used. This type of arrangement is known as smart antenna or MIMO antenna. Since it offers significant increase in data rate and link range without any 
International Journal of Computational Science and Information Technology (IJCSITY) Vol.2, No.2, May 2014

additional bandwidth MIMO has attracted attention in wireless technology predominantly. This could be achieved by spreading the same total transmits power over antennas resulting in improved spectral efficiency and reduced fading. the MIMO Radar has emerged as a trending research area which would increase the detection probability and range resolution far beyond the existing limits. Spatial multiplexing requires MIMO antenna configuration. In spatial multiplexing, a high rate signal is split into multiple lower rate streams and each stream is transmitted from a different transmit antenna in the same frequency channel. Spatial multiplexing can also be combined with precoding when the channel is known at the transmitter or combined with diversity coding when decoding reliability is in trade-off. The primary requirement is that each of the $\mathrm{M}$ elements of the transmitter array must emit signals which are separable in each of the $\mathrm{N}$ receiver elements, allowing the generation of $\mathrm{M} \times \mathrm{N}$ data streams. Because of these properties, MIMO is an important part of modern wireless communication standards such as Wi$\mathrm{Fi}, 4 \mathrm{G}, \mathrm{LTE}$ and Wi-MAX etc.

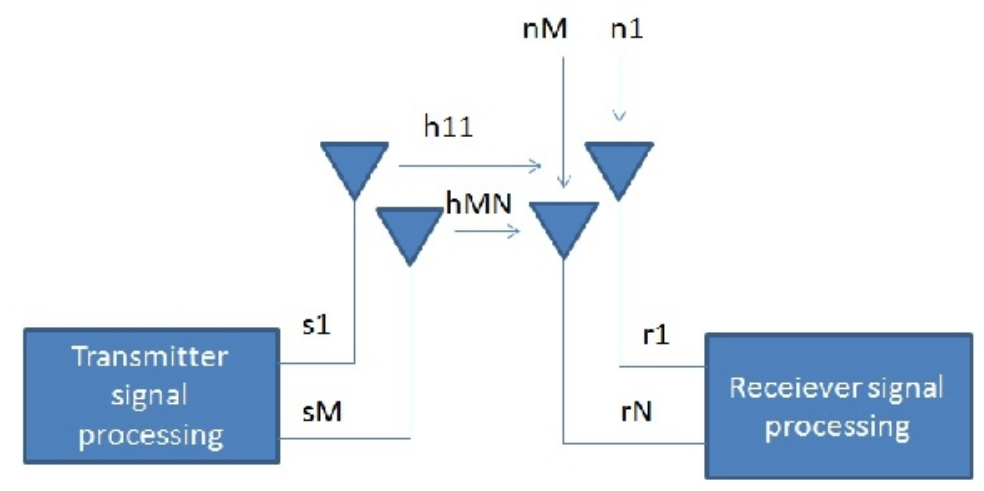

Fig1. M-Transmitter and N-Receiver Multiple antenna configuration.

\section{B. OFDM multi carrier approach}

In digital communication there are two main approaches in which predominantly a waveform is designed. Single carrier is the first among the two does not incorporate frequency diversity. The natural approach, in order to make them suitable for radar measurements, is to optimize their autocorrelation properties and perform time-domain correlation processing in the receiver. The dynamic range, in radar measurements, remains limited, as a result of the only quasi-perfect autocorrelation properties of the resulting transmit sequences. But it had various drawbacks that it requires immense computational effort to calculate the velocity and range in single carrier signals. Also it's affected by fading and Doppler effect. OFDM signals have become a popular choice, in recent wireless communications standards, due to their robustness against fading and multipath propagation effects OFDM, easy synchronization and equalization, and a high flexibility in system design, which allows for easily adapting the system parameters to the given channel characteristics. In contrast to single-carrier waveforms, which are described by a single dimension along the time axis, multicarrier signals also incorporate diversity along the frequency dimension and, hence, can be interpreted as occupying a two dimensional space in time and frequency. This fact offers opportunities for applying sophisticated radar processing algorithms, which go far beyond the classical time domain correlation approach. OFDM signals hence make huge impact in the communication link. The ease of creating a unique MIMO-OFDM have been started much 
International Journal of Computational Science and Information Technology (IJCSITY) Vol.2, No.2, May 2014

earlier and work has been done on this field till now to upgrade the performance of the radar communication both in terms of radar processing and wireless communication [11].

\section{IV.RADAR SENSING}

\section{A.OFDM radar processing}

Radar sensing in general is carried out by transmitting a radio wave and receiving the reflected wave from the target. The reflected wave has information about velocity and range of the reflecting target [12]. The waveform being the most important parameter should suite to both the joint radar and communication application is chosen under optimum constrains placed on it. OFDM rather than a modulation technique is a multiplexing technique, which allows for multiplexing the transmission of symbols over orthogonal subcarriers. The OFDM radar platform is equipped with one transmitter and one receiver for general cases. The radiated signal, which is intended to transmit information to a distant receiver, will at the same time be scattered from objects in the neighborhood and also the receiver that is corresponding to the transmitter will receive these scattered waves. The co-located receiver shares the transmitted information and can use the information for the radar processing. The basic idea of the proposed processing approach consists in evaluating directly the transmitted and received information at the output of the OFDM de-multiplexer before the channel equalization and the decoding is performed [13]. Since all information symbols in one OFDM symbol are transmitted through the channel at different carrier frequencies separated by amount of frequency, the received information symbols can be used in order to perform a channel sensing at discrete frequencies like in stepped frequency radar.

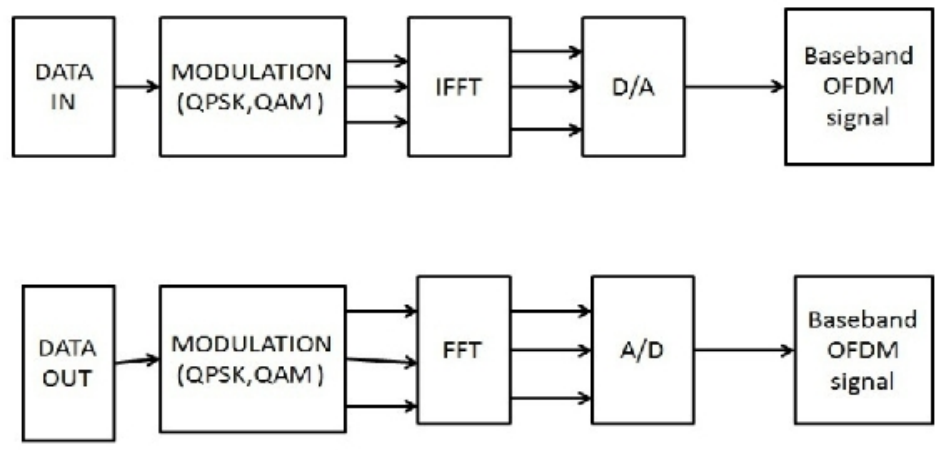

Fig2. OFDM Transmitter and receiver block diagram.

\section{B. Digital beam forming:}

Radar parameters are wide open and few could be found out with the help of the information provided by the reflected wave from the target. But on the other hand computational efforts and signal processing aspects need to be carried out in order to attain certain parameters. Finding the azimuth angle is among them. ie to find the exact 2-D location of the target from the source [14]. Digital; beam forming is similar to that of an MIMO concept in the fact that both the technologies uses multiple antenna techniques. Also the SNR is improved in case of wireless communication 
International Journal of Computational Science and Information Technology (IJCSITY) Vol.2, No.2, May 2014

with the help of MIMO. DBF provides individual antennas in which each consists of receiver and a base band conversion to carry out the processing aspect in radar. There are two main approaches by which digital beam forming could be done effectively. Digital beam forming (DBF) can be realized at sub array level [15]. In the beam forming technique we have adopted the antenna acts in particular direction there by it focuses only on a desired direction. This is termed as digital beam forming due to the digitization and baseband conversion that takes place in the receiver side. To create a constructive and destructive interference pattern a beam former controls the phase and relative amplitude of the signal which in turn changes the directionality of the array when transmitting. In DBF-architecture there are many digital receivers, one own receiver at each of the radiating elements of the antenna. The down-converting to IF-frequency and digitizing the signals is realized at each individual antenna element (or small groups of them). Noise and signal distortion in each receiver are uncorrelated among all receivers. Multiple independent beams steered in all directions can be formed in the digital beam forming processor. The benefits of digital beam forming include improved dynamic range, controlling of multiple beams, better and faster control of amplitude and phase. Since in radar processing the source wave is being transmitted in a rotational path the reflected wave could be sensed based on the angle of arrival or the direction of arrival to the radar transmitting antenna. The first is the Fourier transform based beam forming approach which would consists of simply adding the elements of the rangecompressed signal vector with additional phase shifts. Adaptive beamforming is used to detect and estimate the signal-of-interest at the output of a sensor array by means of optimal (e.g., leastsquares) spatial filtering and interference rejection. To change the directionality of the array when transmitting, a beamformer controls the phase and relative amplitude of the signal at each transmitter, in order to create a pattern of constructive and destructive interference in the wavefront. Most passive radar systems use simple antenna arrays with several antenna elements and element-level digitization.

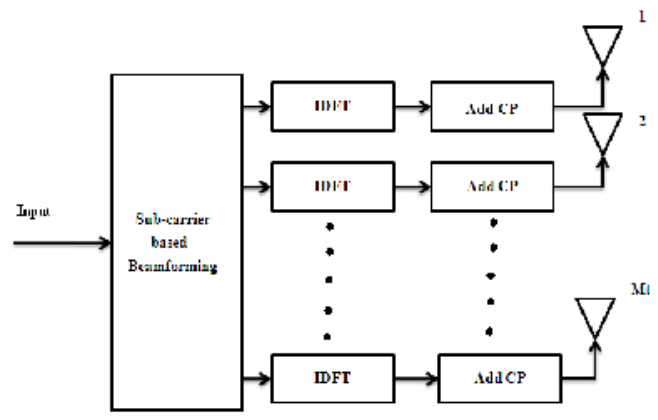

Fig3. Beam forming in OFDM radar transmitter block

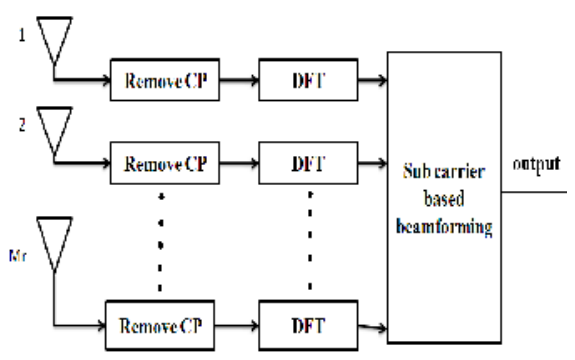

Fig4. Beam forming in OFDM radar receiver block 
International Journal of Computational Science and Information Technology (IJCSITY) Vol.2, No.2, May 2014

\section{V.RESULTS AND DISCUSSION:}

Simulations for the proposed RadCom system is carried out using MATLAB simulation tool and the results were studied. The first and foremost being the waveform design, a simple linear waveform for radar is taken as input and the output waveform is generated. Pulse compression is an important technique involved in radar communication in which the waveform is being modulated first and correlation is done on the receiver.

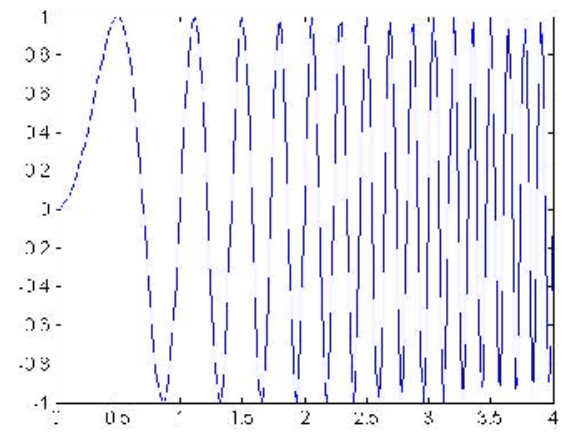

Fig5. Frequency modulated linear waveform used in radar applications.

Frequency modulated wave being the suitable carrier for radar communication is generated. From the above graph it is inferred that increase in the duration of a transmitted pulse increases its energy and improves target detection capability. Conversely, reducing the duration of a pulse improves the range resolution of the radar. Still FM signals prove to be disadvantageous on few aspects. The primary factor is the chirp rate. When chirp rate is low it automatically lowers the symbol rate for the same bandwidth on a dedicated link. Thus OFDM multicarrier signals are chosen considering various advantageous that it provide. Flat fading, bandwidth utilization are important factors that account to the advantages of selecting this OFDM multi carrier waveform. Robust against inter symbol interference (ISI) and fading caused by multipath propagation. OFDM waveforms incorporate frequency diversity, which, through appropriate coding, can be exploited for increasing the robustness of the transmission. These techniques offer a number of advantages, such as the availability of processing gain at the receiver, a whitening of the signal spectrum, and a reduction of the power spectral density. A typical example is m-sequence radars, which employ pseudorandom $\mathrm{m}$-sequences for discrete phase modulation, in order to create a noise-like transmit signal with good autocorrelation properties. The applicability of multicarrier waveforms for radar purposes has been demonstrated as well.

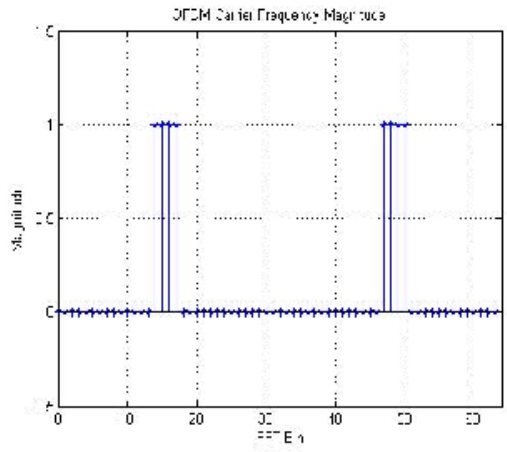

Fig6. OFDM carrier frequency magnitude plot

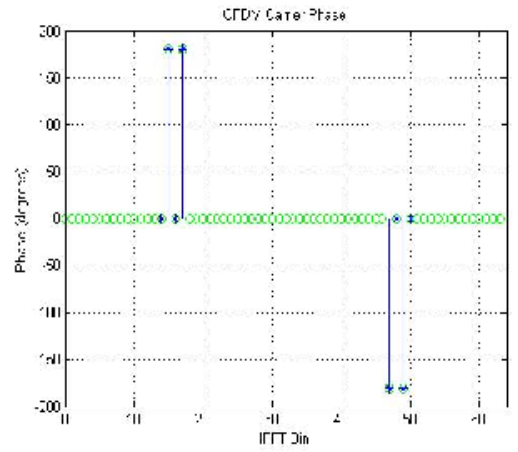

Fig7. Plot for OFDM crarrier phase 


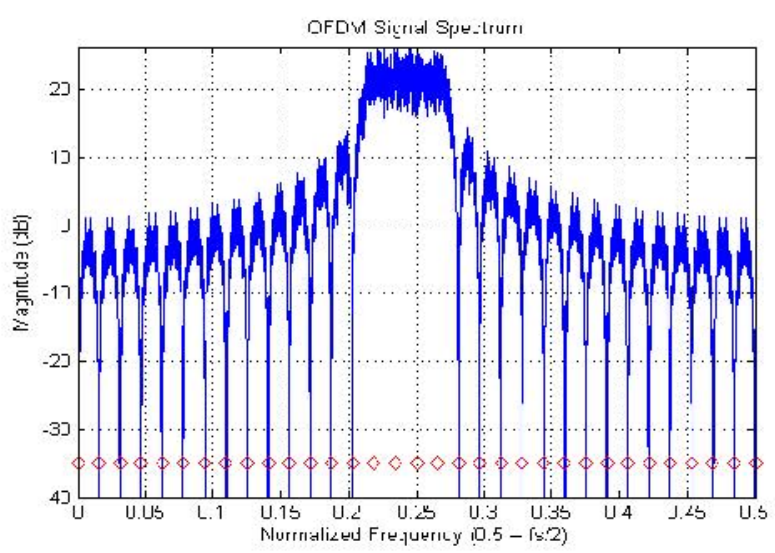

Fig8. OFDM signal spectrum

From the above graph it can be inferred that an OFDM multi carrier signal is more advantageous in terms of spectrum efficiency and subcarriers are orthogonal to each other. The OFDM carrier frequency signal is transmitted and reflects from the target. It contains information about the target like velocity with which it's travelling, azimuth angle etc. From communication perspective to improve the performance, MIMO or smart antenna concept used in the work which gives much better results in terms of data rate and bit error rate. Quadrature Amplitude Modulation (QAM) is adopted with the assumption that channel knowledge is known at the transmitter and noise is modelled as Gaussian random noise with zero mean and variance $N_{0}$. Advantage of OFDM is that the equalization complexity at the receiver is reduced dramatically. These features of OFDM are very attractive that when implemented along with multiple-input multiple-output system there is a drastic improvement in the system performance. MIMO systems exploit space diversity and OFDM exploits frequency diversity when these techniques are combined the system performance improves. MIMO has other advantages like performing beamforming when the channel information is known at the transmitter, space time coding such as Alamouti coding can be used to exploit time diversity when the channel state information is not known at the transmitter.

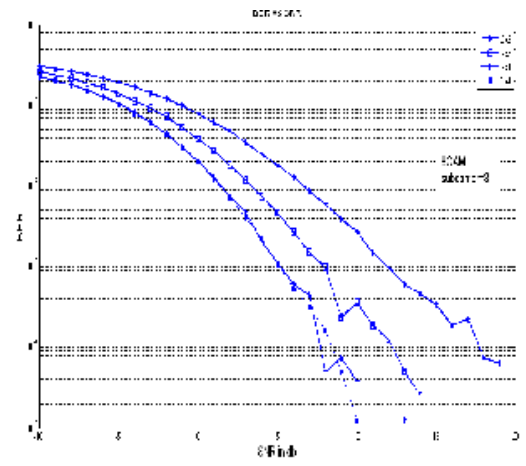

Fig9. BER vs. SNR plot for MIMO with 8-QAM

From the above graph it is inferred that the signal to noise ratio increases for a MIMO configuration and as the number of receiver is increased it decreases the bit error rate. In this case 
International Journal of Computational Science and Information Technology (IJCSITY) Vol.2, No.2, May 2014

we have inferred that a 2 x 4 system ( 2 transmitters and 4 receivers) offers low BER than other systems. The subcarrier level is varied and the resulting output is generated.

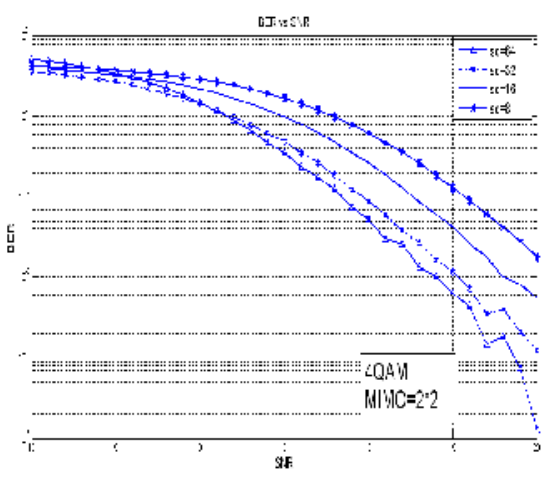

Fig10. BER vs. SNR plot for MIMO-OFDM at various subcarrier levels.

The graph for SNR vs. BER is given and it shows that as the subcarrier is increased it offers low bit error rate. The above result is simulated for a $2 \times 2$ MIMO system with increased subcarrier that enables the ease of MIMO-OFDM radar processing. Digital beam forming is carried out with the help of Fourier transform approach and the result for the OFDM input waveform is analyzed. The various transmitter and receiving configuration is analyzed and beam forming is added to the normal OFDM system. High data rate and highly reliable communication is the basic requirement for nearly every wireless communication system. OFDM uses frequency diversity where each data symbol is modulated using orthogonal carriers and hence interference is reduced. Since a huge bandwidth is chopped into small bandwidths, the symbol duration of each symbol increases. The increased symbol duration is the requirement to reduce the multipath spread of the symbol. Beamforming is applied to separate sub-carrier to reduce the interference among the carriers. Then IDFT is applied to convert the symbol from time domain to frequency domain to analyze symbol characteristics. Cyclic prefix is added in order to reduce the inter symbol interference (ISI). All the symbols are transmitted through $1^{\text {st }}$ antenna, then it switches to $2^{\text {nd }}$ antenna and so on. Reverse operation takes place in receive beamforming

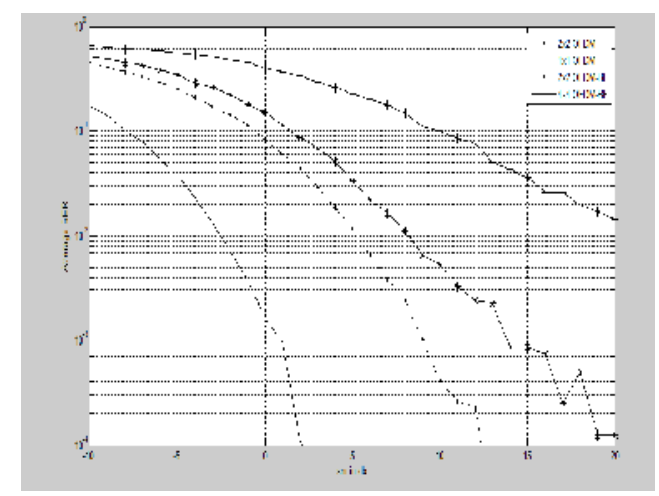

Fig11. Plot of a general OFDM system and a beam form included system.

From the above plot is ensures that an OFDM system included with beam forming performance better than a general OFDM system. 

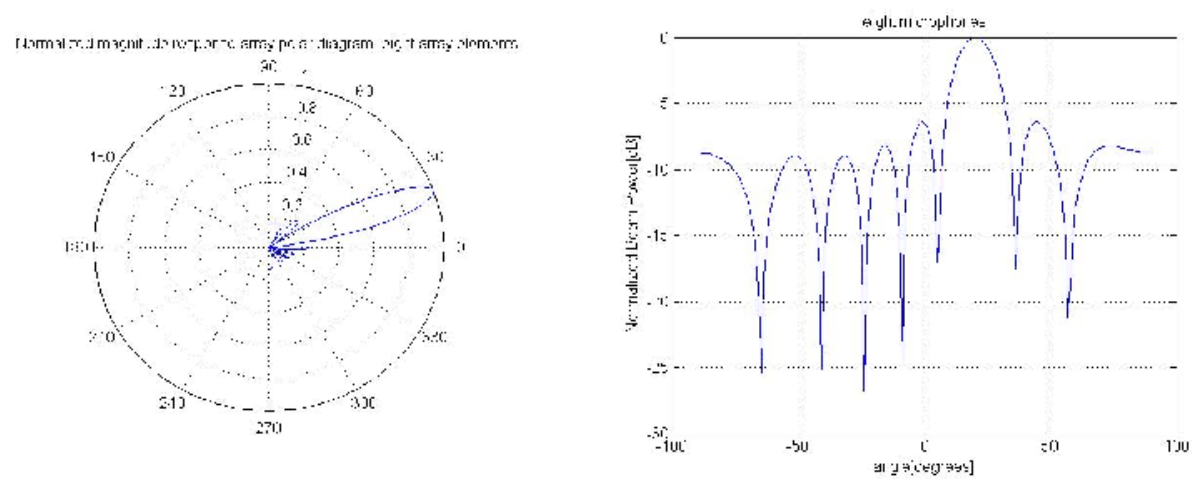

Fig12. Beamforming plot to detect angle of the target

From the above graph it is inferred that when a target is placed at a certain angle with the help of beamforming the angle is found out by forming a virtual beam towards the particular direction. Thus the simulation results give a complete idea of how a RadCom system would behave with the applied properties. The digital beam forming is an integration between antenna technology and digital technology. A beamformer is a processor used in conjunction with an array of antennas to provide a versatile form of spatial filter. In digital beamforming the operations of phase shifting and amplitude scaling for each antenna element, and summation for receiving, are done digitally. Either general-purpose DSP's or dedicated beamforming chips are used. Digital processing requires that the signal from each antenna element is digitized using an A/D convertor. Since radio signals above short wave frequencies $(>30 \mathrm{MHz})$ are too high are directly digitized at a reasonable cost, digital beamforming receivers use analog "RF translators" to shift the signal frequency down before the A/D convertors. Once the antenna signals have been digitized they are passed to "digital down convertors" that shift the radio channels center frequency down to $\mathrm{OHz}$ and pass only the bandwidth require for one channel. The down convertors produce a "quadrature" baseband output at a low sample rate. For beamforming the complex baseband signals are multiplied by the complex weights to apply the phase shift and amplitude scaling required for each antenna element.

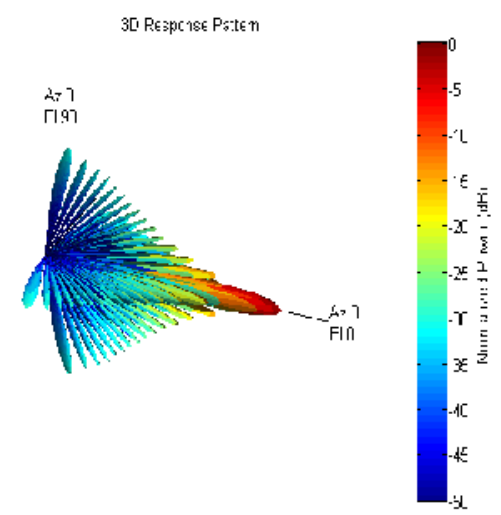

Fig13.rectangular array pattern

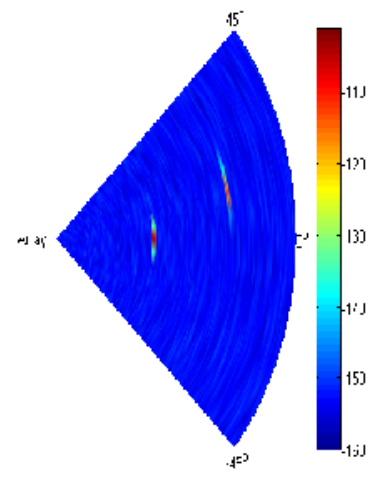

Fig14.radar image with two reflected target 
International Journal of Computational Science and Information Technology (IJCSITY) Vol.2, No.2, May 2014

A 900-element rectangular array is used in this mono static radar. Rectangular array pattern is taken for beamforming and azimuth angle is found out in the desired direction. Two targets are considered and the signal is sent. The radar image is obtained by considering all the parameters that suite's for a radar environment. From the scan map, we can clearly see two peaks. The close one is at around 0 degrees azimuth, the remote one at around 10 degrees in azimuth.

\section{VI.INFERENCE FROM THE RESEARCH}

Radar being the forerunner in the field of communication will have a huge impact when all images could be transmitted with the remote user there by the range could be extended far beyond the existing limits. In this paper we have discussed the waveform design that perfectly suited for the combined operation of radar sensing and wireless communication. We have placed certain constrains on the waveform design and it has been designed to meet the requirements relating to spectral bandwidth efficiency. OFDM multicarrier waveforms being more advantageous than single carrier in all aspects perfectly matches to the designed RADCOM system. We have also put forth the multiple antenna concepts by which both the applications could be benefited. In terms of communication MIMO is used for improving the data rate there by the signal to noise ratio increased predominantly. Similarly in case of radar, digital beam forming can be implemented to find the azimuth angle. The operation of the system takes place in a perfectly matching bandwidth, the $24 \mathrm{GHz}$ ISM band. Thus the combination of radar sensing and wireless communication enables the radar range to extend far beyond the limits and also the radar image can be shared with the remote users. The implementation of the proposed RadCom system would replace the existing hardware complexity and reduce the cost-effectiveness.

\section{VII.REFERENCES}

[1] M. I. Skolnik, Radar Handbook, 3rd. New York: McGraw-Hill, 2008.

[2] G. N. Saddik, R. S. Singh, and E. R. Brown, "Ultra-wideband multifunctional communications/radarsystem,” IEEE Trans. Microw. Theory Techn., vol. 55, no. 7,pp. 1431-1437, Jul. 2007.

[3] S. D. Blunt, P. Yatham, and J. Stiles, "Intrapulseradar-embedded communications," IEEE Trans. Aerosp.Electron. Syst., vol. 46, no. 3, pp. 1185-1200,Jul. 2010.

[4] B. J. Donnet and I. D. Longstaff, "Combining MIMO radar with OFDM communications," in Proc. 3rd European Radar Conf. 2006,Sep. 2006, pp. 37-40.

[5] A. De Maio, S. De Nicola, Yong-Wei Huang ; Shu-Zhong Zhang, A. Farina, "Code design to optimize radar detection performance under accuracy and similarity constraints" IEEE Trans. Signal Processing,Vol. 56, No. 11, pp. 5618-5629, 2008.

[6] A. De Maio, S. De Nicola, Y. Huang ; Z. Luo, S. Zhang, "Design of phase codes for radar performance optimization with a similarity constraint" IEEE Trans. Signal Processing, Vol. 57, No. 2, pp. 610-621,2009.

[7] B. Jiu, H. Liu, L. Li, S. Wu, "Waveform design for broadband radar based on phase modulated signal," Journal of Electronics \& Information Technology, Vol. 30, No. 9, pp. 2038-2041, 2008.

[8] C. Sturm, M. Braun, T. Zwick, andW. Wiesbeck, "A multiple target Doppler estimation algorithm for OFDM based intelligent radar systems" in Proc. European Radar Conf. 2010, Paris, France, Sep. 2010.

[9] C. Sturm, M. Braun, T. Zwick, and W. Wiesbeck, "Performance verification of symbol-based OFDM radar processing" in Proc. IEEE 2010 Radar Conf., Washington, DC,May 2010.

[10] P. van Dorp, R. Ebeling, and A. G. Huizing, "High resolution radar imaging using coherent multiband processing techniques," in Proc .IEEE Radar Conf., Washington, DC, May 2010, pp. 981-986

[11] C. Sturm, E. Pancera, T. Zwick, and W. Wiesbeck, "A Novel Approach to OFDM Radar Processing," Radar Conference, IEEE, May 2009. 
International Journal of Computational Science and Information Technology (IJCSITY) Vol.2, No.2, May 2014

[12] M. Braun, C. Sturm, and F. K. Jondral, "Maximum Likelihood Speedand Distance Estimation for OFDM Radar," Radar Conference, IEEEInternational, 2010.

[13] G. Lellouch, P. Tran, R. Pribic, and P. van Genderen, “OFDM waveforms for frequency agility and opportunities for Doppler processing in radar," in Proc. IEEE 2008 Radar Conf. (RADAR 2008), May 26-30, 2008, pp. 1-6.

[14] Krieger, G., Gebert, N., and Moreira, A. Multidimensional waveform encoding: A new digital beam forming technique for synthetic aperture radar remote sensing. IEEE Transactions on Geoscience and Remote Sensing, 46.1(2008), 31-46

[15] Chen, J., J. Gao, Y. Zhu, W. Yang, and P. Wang, "A novel image formation algorithm for highresolution wide-swath spaceborne SAR using compressed sensing on azimuth displacement phase center antenna," Progress In Electro magnetics Research, Vol. 125, 527,543, 2012.

\section{Authors}

A.Suban, received B.E in the department of Electronics and Communication Engineering from Anna university, Chennai and M.E in the discipline of Wireless Technology from Thiagarajar College of Engineering, Madurai in 2011. He is currently working as Assistant Professor in the Department of Electronics and Communication Engineering, Velammal College of Engineering and Technology, Madurai-625009, Tamil Nadu, India. His area of interest in Signal processing mainly focused on MIMO techniques with beamforming, OFDM and power control techniques.

G.Thanga Balaji, pursuing Bachelor of engineering in Velammal College of Engineering and Technology, Madurai-625009. H is area of interst is signal processing.
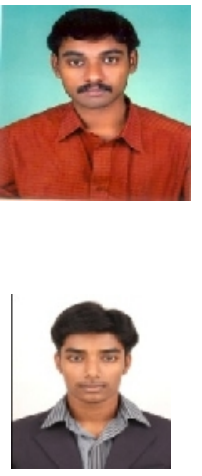

C.Kaleeswaran, pursuing Bachelor of engineering in Velammal College of Engineering and Technology, Madurai-625009. His area of interst is signal processing.

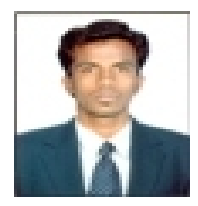

H.Mani Aravinth.,pursuing Bachelor of engineering in Velammal College of Engineering and Technology, Madurai-625009. His area of interst is signal processing.

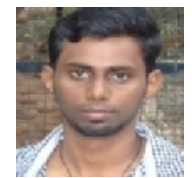

